\title{
Development of a TPC detector for the ALICE Experiment.
}

\author{
Joachim Bächler ${ }^{\mathrm{b}}$ Juraj Bracinik $^{\mathrm{a}}$ Hans Gerhard Fischer ${ }^{\mathrm{b}}$ \\ Marcel Flammier ${ }^{\mathrm{b}}$ Rudolf Janik ${ }^{\mathrm{a}}$ Jean Claude Legrand ${ }^{\mathrm{b}}$ \\ Luciano Musa ${ }^{\mathrm{b}}$ Miroslav Pikna ${ }^{\mathrm{a}}$ Branislav Sitar ${ }^{\mathrm{a}}$ \\ Piotr Szymanski b,c
}

Presented by Juraj Bracinik.

For the ALICE Collaboration.

${ }^{a}$ University of Bratislava, Bratislava, Slovakia.

${ }^{\mathrm{b}}$ CERN, Geneva, Switzerland.

${ }^{\mathrm{c}}$ Institut for Nuclear Studies, Warsaw,Poland.

\begin{abstract}
A proportional chamber with ring cathode readout is foreseen for the ALICE Time Projection Chamber. It offers low gas gain operation, light-weight construction and good pulse shape. The Tape Automatic Bonding (TAB) process makes it possible to mount the VLSI analog front end electronics directly on the back face of the multilayer board carrying the ring cathode elements, yielding high channel density. At the same time, simulation work is done. Construction and first results will be presented.
\end{abstract}

\section{Introduction.}

ALICE, the dedicated heavy-ion experiment to operate at the LHC collider [1] will feature a Time Projection Chamber (TPC) as central tracking device. This TPC will be of the cylindrical shape already used in various preceding collider experiments. Its dimensions are large, but not unusual for such applications(inner radius $90 \mathrm{~cm}$, outer radius $250 \mathrm{~cm}$, total length $500 \mathrm{~cm}$ subdivided into two drift spaces of $250 \mathrm{~cm}$ drift length from a central high voltage plane). It is, however, a rather challenging device due to its experimental boundary conditions: 
- operation in a low magnetic field of $0.2 T$ for tracking and identification of particles down to transverse momenta of $100 \mathrm{MeV} / \mathrm{c}$

- operation in very large track densities reaching $3 \times 10^{4}$ tracks per event in the drift volume

- as a consequence, very high electronics density with $5 \times 10^{5}$ channels corresponding to $0.6 \mathrm{~cm}^{2}$ pad surface

- long drift time of $100 \mu \mathrm{s}$ with $10^{3}$ time samples in steps of $100 \mathrm{~ns}$

- high expected current load of about $1 \mu \mathrm{A} / \mathrm{m}^{2}$ detector surface due to tracks and background penetrating the readout chambers

\section{Working environment.}

ALICE is foreseen to operate with a variety of ion species as well as with proton beams.

In $\mathrm{Pb}-\mathrm{Pb}$ running, event rates will reach $10^{4}$ minimum bias events per second. A few percent of this rate corresponds to central collisions which will be trigger-selected. For these central events, about $8 \times 10^{3}$ charged particles per unit rapidity are to be expected. This leads to $3 \times 10^{4}$ tracks in the drift volume of which about half are reconstructed with full length for $d E / d x$ measurement. A substantial event-correlated background is created by secondary interactions and backscattering in the detector environment. Under these conditions the accumulation of positive ionization space charge is becoming a major concern. Neon gas mixtures with low specific ionization and increased ion drift velocities have therefore been selected.

In proton running, the maximum luminosity will be limited to $10^{30} 1 / \mathrm{cm}^{2}$ s i.e. four orders of magnitude below the maximum LHC luminosity. This corresponds to $10^{5}$ interactions per second or a pile-up of 10 events per readout cycle.

Even without accounting for event-correlated and accelerator-induced backgrounds, these working conditions would lead, with classical readout chamber design, to DC currents between 0.1 and $1 \mu A$ per $m^{2}$ detector surface. With such loads, long-term stability problems induced by cathode and anode ageing processes have to be expected.

This led to the development of a modified readout chamber design featuring low active chamber volume and increased signal coupling, inspired by the classical proportional tube geometry. 


\section{Ring cathode chambers.}

By folding the cathode readout elements (pads) around the amplifying sense wires, see fig. (1), an increase of signal coupling to the pads of about a factor of four, as compared to the standard flat pad plane arrangements, can be achieved. Integrating the gating elements mechanically into the this ring structure, fig. (1), the active chamber volume below the gating plane can be reduced by about a factor of two. An eight-fold overall reduction of the current load due to particles penetrating the readout plane can therefore be expected. In addition, the elimination of three wire planes (gating grid, Frisch grid and field wires) alleviates constructional and geometrical design problems.

Two techniques for ring cathode production are currently under study: bending of thin $\mathrm{Cu}$ sheets and stamping (fine cold forging) of $\mathrm{Al}$ pads.

In adition, the time dependence of the induced signal, fig. (2) changes from the substantial undershoot behaviour observed in flat cathode arrangements, into $1 / t$ tail typical of proportional tubes. This allows effective electronics tail supression techniques to be applied. In view of the very high occupation density of the electronics reaching $30 \%$ at the inner radius, base line stability becomes indeed a major concern.

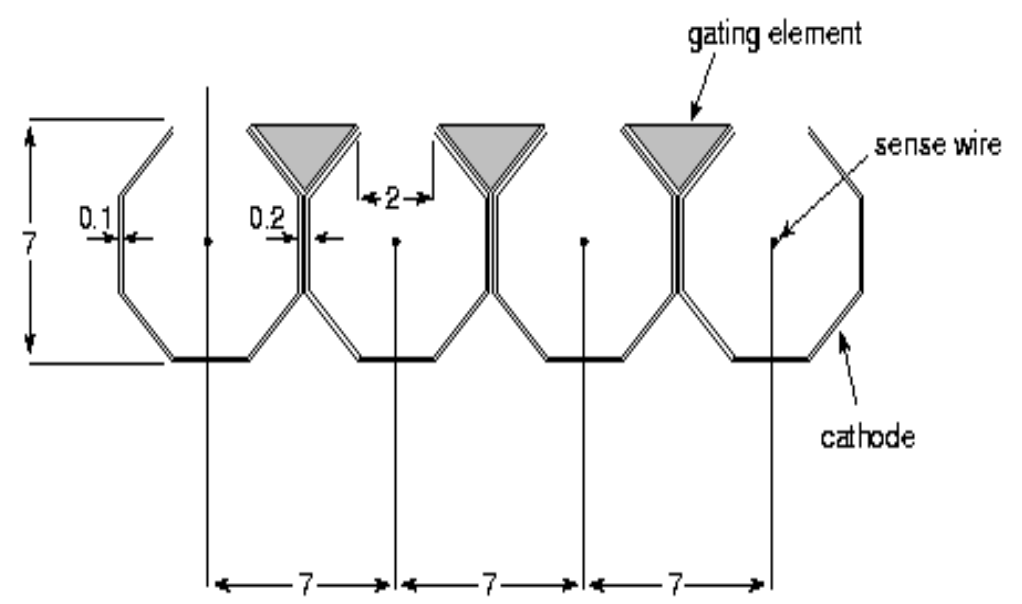

Fig. 1. RCC readout of TPC.

\section{Front-end electronics.}

A custom bipolar preamplifier/shaper circuit with four electronics channels per chip has been designed at CERN [5] and realised in Harris UHF technology [2]. It performs base-line restoration to better than $0.1 \%$ of peak pulse height. 

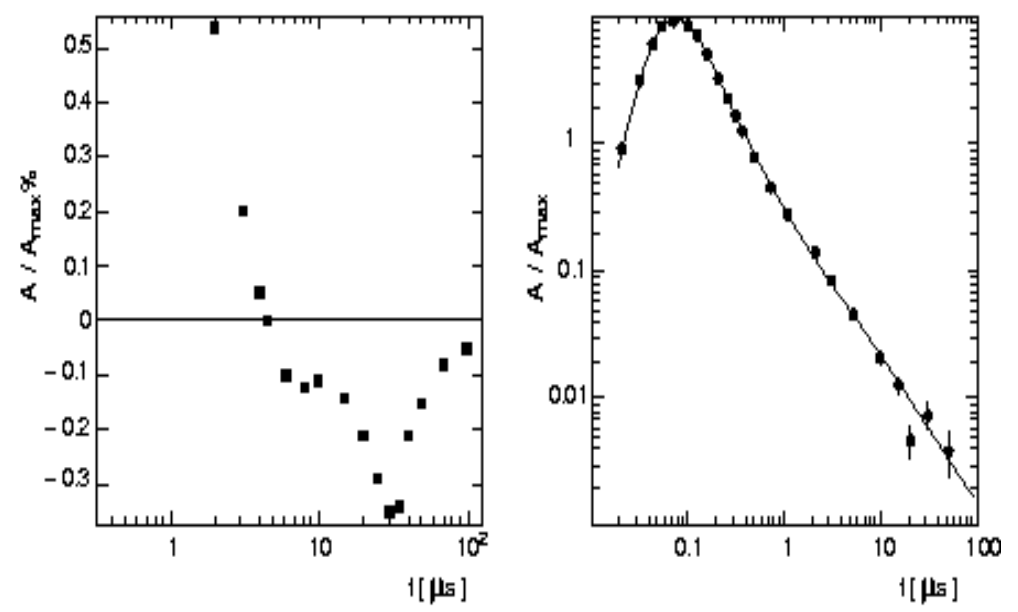

Fig. 2. Measured pulse shape from standard TPC and RCC.

The four-channel dies are bonded directly onto the backplane of the readout chamber, using the Tape Automatic Bonding (TAB) process developed by Dassault Electronics [4]. The TAB technology allows full dynamical functionality testing of each die before transfer to the multilayer PC board which carry about 150 chips corresponding to an electronics density of 1.3 channels per $\mathrm{cm}^{2}$.

The signal from the preamplifier/shaper is sampled and digitized with a frequency of $10 \mathrm{MHz}$ by fast $\mathrm{ADC}$ circuits with a dynamical range of $10 \mathrm{bits}$. This dynamical range is necessary owing to the large swing in $d E / d x$ to be expected in the low-momentum range of ALICE and to the necessary tracking and $d E / d x$ performance (see below).

Several types of commerciayl available ADC circuits were tested $[3,6,7]$ using output pulse shapes from proportional chambers. Power consumption of these devices is a major concern as they have to be mounted directly onto the readout chamber in the final TPC design. In a prototype test foreseen for fall 1998, the ADC's are mounted at about $2 m$ distance from the detector and connected via coaxial cables of only 500 $\mu \mathrm{m}$ diameter (Piko-Coax, [8]).

\section{$5 \quad$ Tracking performance.}

The ALICE TPC will have to perform event pattern recognition and track extrapolation towards the inner tracking system and towards the outer detectors. In addition to careful minimisation of track distortions and alignment errors, the space resolution has to be well controlled. Detailed simulations have been performed and compared to measurements in the Na49 TPC detector at CERN [9]. We will concentrate here on the understanding of the first two terms in the usual expansion of the TPC space resolution (see e.g. [10]). 


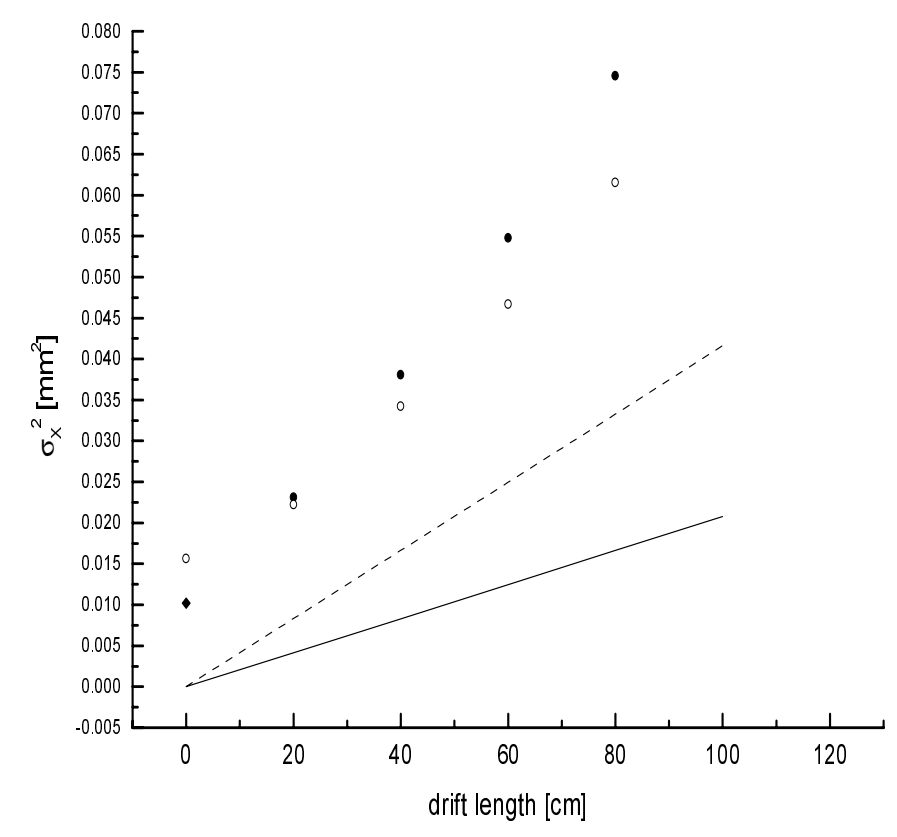

Fig. 3. Space resolution from data and simulation.

$$
\sigma_{C O G}^{2}=\sigma_{0}^{2}+\frac{D_{T}^{2} L_{\text {drift }}}{N_{\text {electrons }}}+\text { angular terms }
$$

We have shown that the sigmazero term is basically determined by electronics noise and zero supression treshold applied to the readout data. It can be minimized by rising the measured pulseheight with respect to the rms noise as much as compatible with dynamic range and stable chamber operation.

The length-dependent diffusion term has been shown to be influenced by gas gain fluctuations in the avalanche process at the sense wire: a factor of 2 has to be introduced in the squared resolution,

$$
\sigma_{C O G}^{2}=\sigma_{0}^{2}+\frac{2 D_{T}^{2} L_{\text {drift }}}{N_{\text {electrons }}}+\text { angular terms }
$$

in order to take care of this effect, the factor following from the exponential shape of the avalanche pulse height distribution. It should be noted that this effect acts only on the center-of-gravity precision and not on the rms width of the charge distributions. With this correction, the measured TPC resolution can be well predicted by Monte Carlo methods, as shown in fig. (3). Here lines correspond to diffusion and modified diffusion term, open bullets to the simulation and filled bullets to the data. 


\section{References}

[1] ALICE, Technical Proposal CERN/LHCC 95-71, ISBN 92-9083-077-8.

[2] Harris Semiconductors, represented by SdM Microelectronique Charleroi (B).

[3] Harris ADC \#HI 5710.

[4] Dassault Electronique, StCloud (F).

[5] J.C. Legrand, CERN/EP Division.

[6] Philips ADC \#TDA 8766.

[7] Analog Devices ADC \#AD 9200.

[8] Pico-coax, AXON CABLE, Leonberg.

[9] J.Bracinik,H.G.Fischer, to be published.

[10] Particle Detection with Drift Chambers, W.Blum,L.Rolandi, SpringerVerlag, 1993. 